\title{
Neighborhood Boundaries and Violent Crime. An Introduction to Boundary Detection Methods in $\mathrm{R}^{*}$
}

\author{
Joscha Legewie
}

April 17, 2018

Neighborhood boundaries are a defining aspect of highly segregated urban areas. Yet, few studies examine the particular challenges and spatial processes that occur at the bordering region between two neighborhoods. This guide introduces readers to different methods to measure neighborhood boundaries. It illustrates the use of boundary detection methods in the social science. The analysis is loosely based on Model II in Table 2 of Legewie (2018).

The analysis estimates the relationship between violent crimes and racial neighborhood boundaries in Chicago. It is based on the census tract level and uses geo-coded, incident-level crime data from the Chicago Police Department from 2011 combined with data on from the 2010 Census and the 2007-2011 5-year estimates from the American Community Survey (ACS). The section "Boundary Detection" below illustrates areal wombling as a boundary detection approach designed for areal data (see Legewie 2018 for details).

Edit: Updated to run with recent $\mathrm{R}$ versions and focus on Areal wombling.

\section{Required packages}

The code requires the packages dplyr (version 1.0.7), purrr (version 0.3.4), readr (version 2.0.2), tidyr (version 1.1.4), tibble (version 3.1.5), sf (version 1.0-0), MASS (version 7.3-54), stargazer (version 5.9.9), scales (version 1.1.1), matrixStats (version 0.59.0), sp (version 1.4-5), tidycensus (version 1.3), and BoundaryDetection (version 0.2). More recent versions might work as well. Many of these packages can be installed with install.packages ("tidyverse").

library ("MASS")

library("sp")

library ("stargazer")

library ("sf")

library ("matrixStats")

library ("tidyverse")

library ("scales")

library ("tidycensus")

library ("BoundaryDetection")

Setting your Census API key The R package tidycensus uses the Census API to download census data. Accessing the Census API requires a key. You have to request a key from here and set it $\mathrm{R}$ with the command tidycensus: :census_api_key("YOUR KEY", install = TRUE).

*Please direct correspondence to Joscha Legewie, Yale University, Department of Sociology, P.O. Box 208265, New Haven CT 06520-8265 (joscha.legewie@yale.edu). 


\section{Download and Recode Census Data}

We begin by downloading 2010 decennial census data on the census tract level (chi_sf1) and 2007-2011 ACS 5 -years estimates (chi_acs) for Cook County, Illinois. The tidycensus package makes it easy to download and work with census data using the Census API. At a later state of the analysis, we restrict our data to Chicago by excluding census tracts that are in Cook County but outside of the city limits.

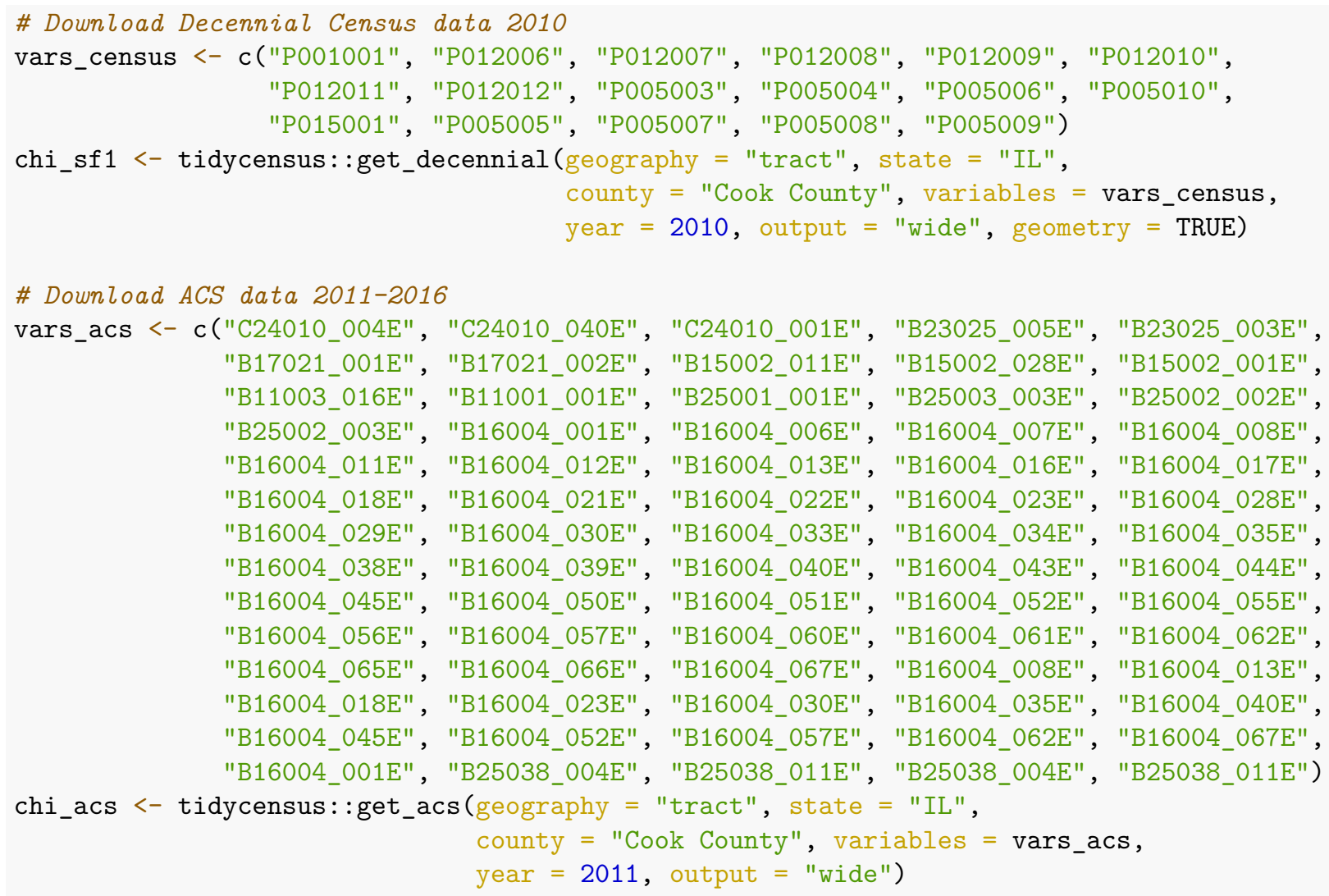

After downloading the data, we recode both the decennial census and ACS data and merge the two data sets.

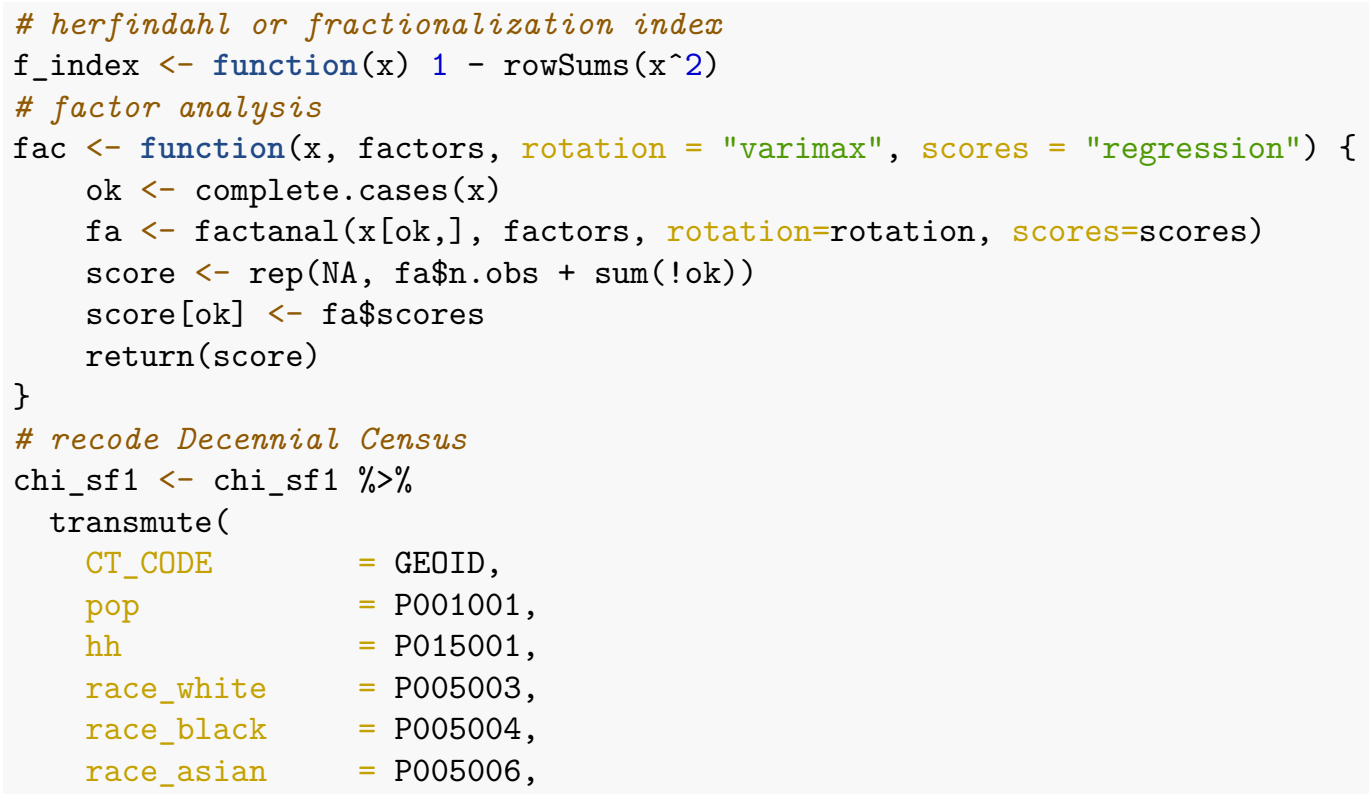




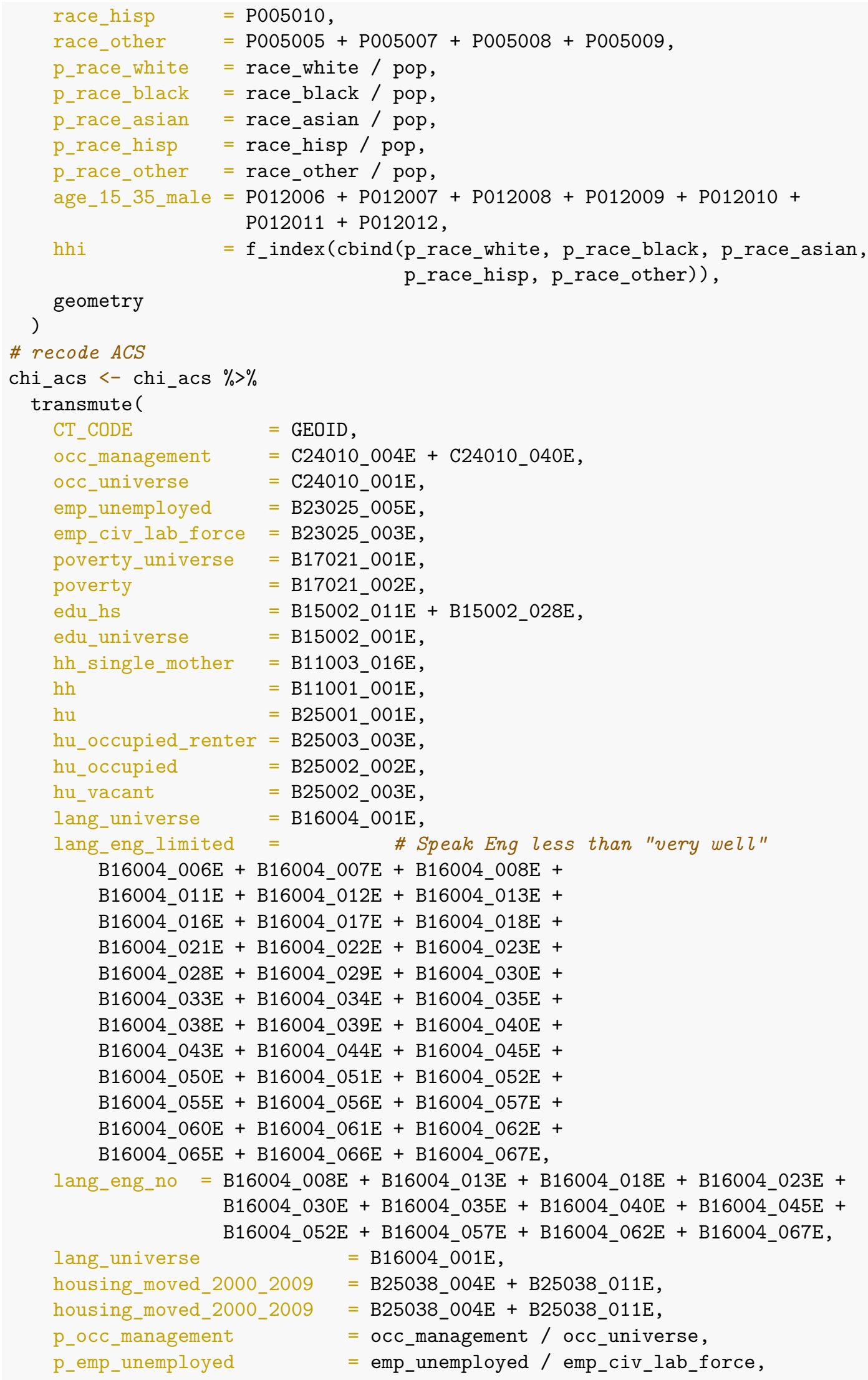




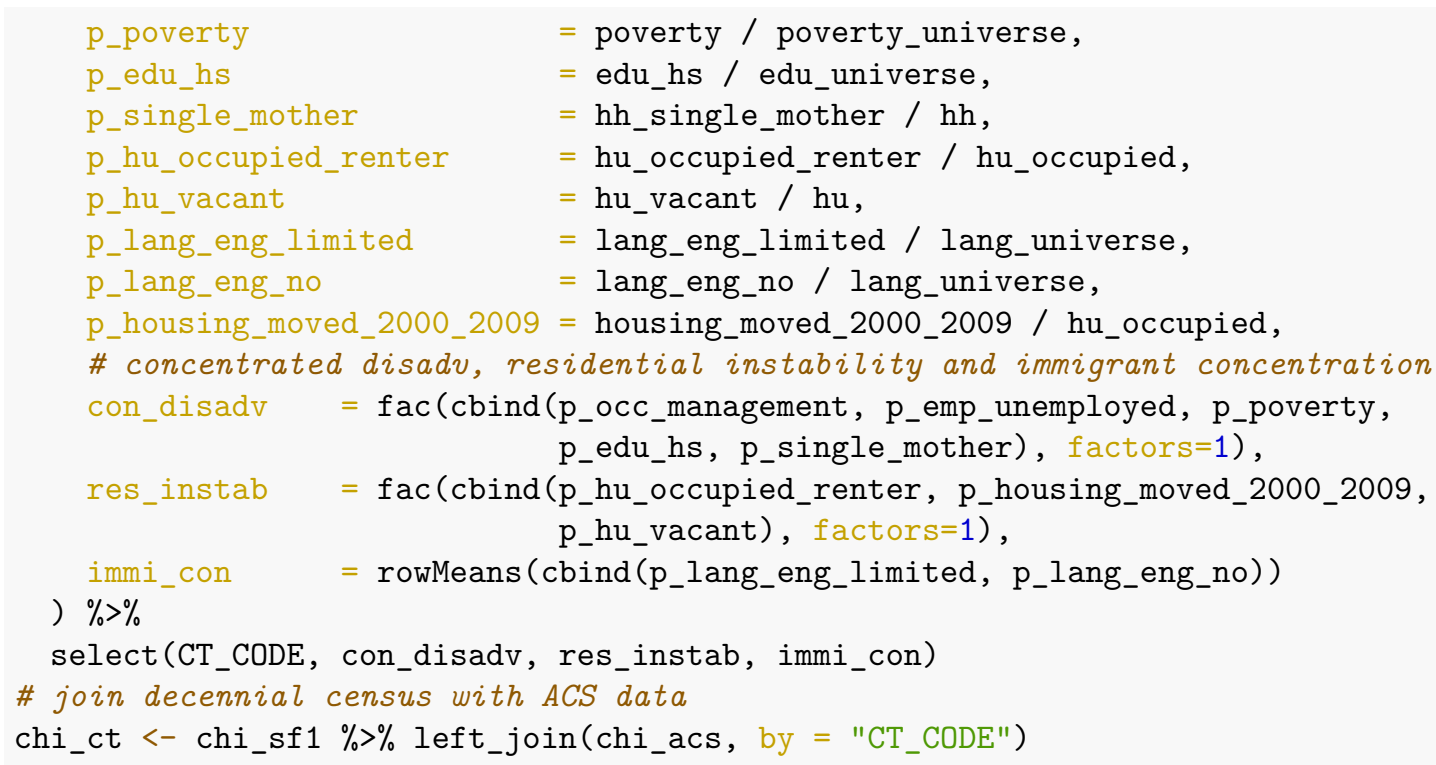

\section{Merging with crime data}

Incident-level crime data from Chicago is available from the Chicago Data Portal. Here we use an aggregated version of the data that includes the number of violent crime in each census tract in 2011.

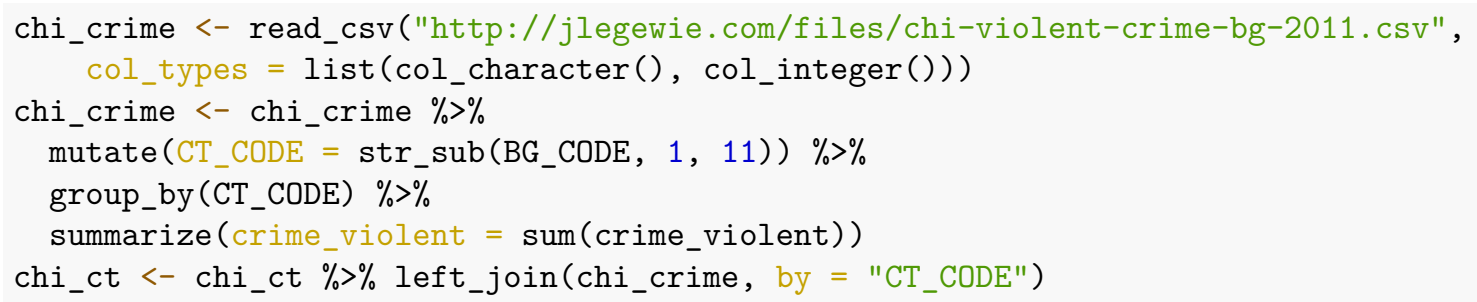

Crime data is only available for Chicago and not other parts of Cook County. Accordingly, the variable crime_violent is missing for census tracts that fall outside of the city limits. We use this fact to restrict our date to Chicago.

chi_ct <- chi_ct $\%>$ filter (complete.cases (crime_violent))

\section{Boundary Detection}

The main independent variable is based on racial neighborhood boundaries on the census tract level. Most existing boundary analysis methods are designed for point- (or raster-) level data. Point-specific data consists of points with known coordinates (latitude and longitude) such as incident-level crime data with precise information on the location of the crime. Neighborhood research based on contemporary data, however, generally relies on aggregated census data for geographical regions such as census blocks, tracts, counties or zip codes. Areal data refers to aggregated information for some region (e.g. census tracts, counties, or zip codes) with information on the location and shape of these regions (spatial polygons often in the form of shapefiles). Examples include the number of crimes in a certain time-interval, the racial composition, or poverty rate across census tracts in the United States.

Areal wombling Areal wombling is a boundary detection approach designed for areal data (see Legewie 2018 for details). It is based on the idea that two adjacent areas are separated by a boundary if the difference 
between the two areas in some response variable exceeds a threshold. This difference between all pairs of adjacent areas is estimated based on some distance metric such as Euclidean distance, squared Euclidean distance, or Manhattan distance. More formally, consider an areal map with $i=1, \ldots, N$ regions, a response variable $Y_{i}$ (e.g., proportion of African-American residents, poverty rate or number of violent crimes), and an adjacency matrix $A$ that is defined as $a_{i j}=1$ when $i$ and $j$ are adjacent and 0 otherwise. For each pair of adjacent regions, the boundary likelihood value (BLV) or just the boundary value is defined as $D_{i j}=\left\|Y_{i}-Y_{j}\right\|$ where $\|\cdot\|$ is a distance metric. High boundary values reflect a large difference in the response variable between adjacent areas indicating that the transition from $i$ to $j$ is part of the boundary.

The package BoundaryDetection allows us to calculate the boundary value $D_{i j}$ for each pair of adjacent areas using the function areal_wombling. The first argument is the spatial geometry object with attribute data. sf and SpatialPolygonsDataFrame objects are both supported. The second argument is a vector of variable names for which we want to calculate the boundary value.

vars <- c("p_race_white", "p_race_black", "p_race_hisp", "p_race_asian")

bdr <- areal_wombling(chi_ct, vars)

The output bdr is a SpatialLinesDataFrame. It includes spatial lines for each bordering line segment between adjacent areas and the corresponding boundary value for each variable such as p_race_white_blv, p_race_black_blv or p_race_hisp_blv. Let us plot the result. The figure shows census data for the proportion of African-American residents across census block groups on the left side and the spatial bordering lines on the right side. Thicker lines indicate higher boundary values.

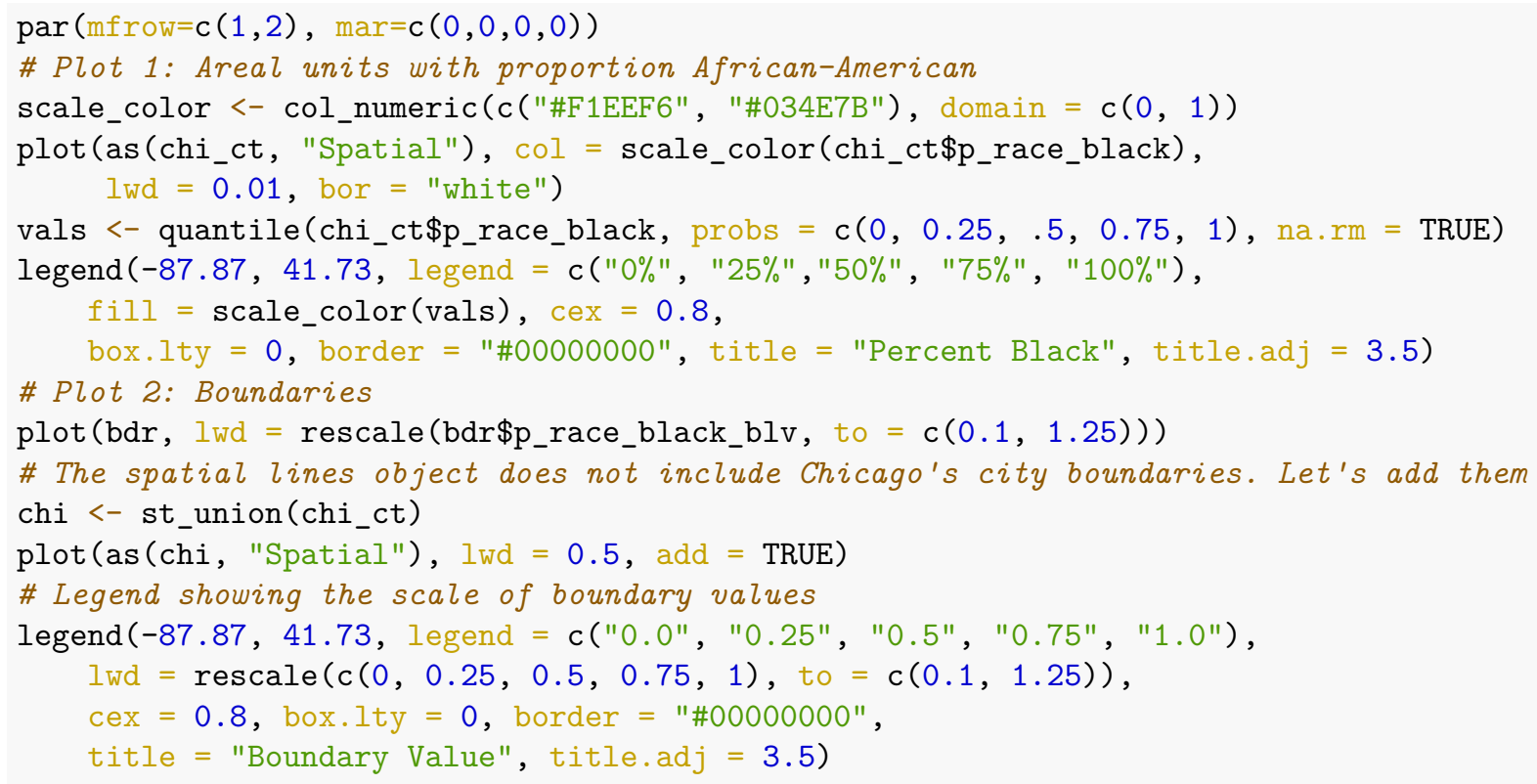



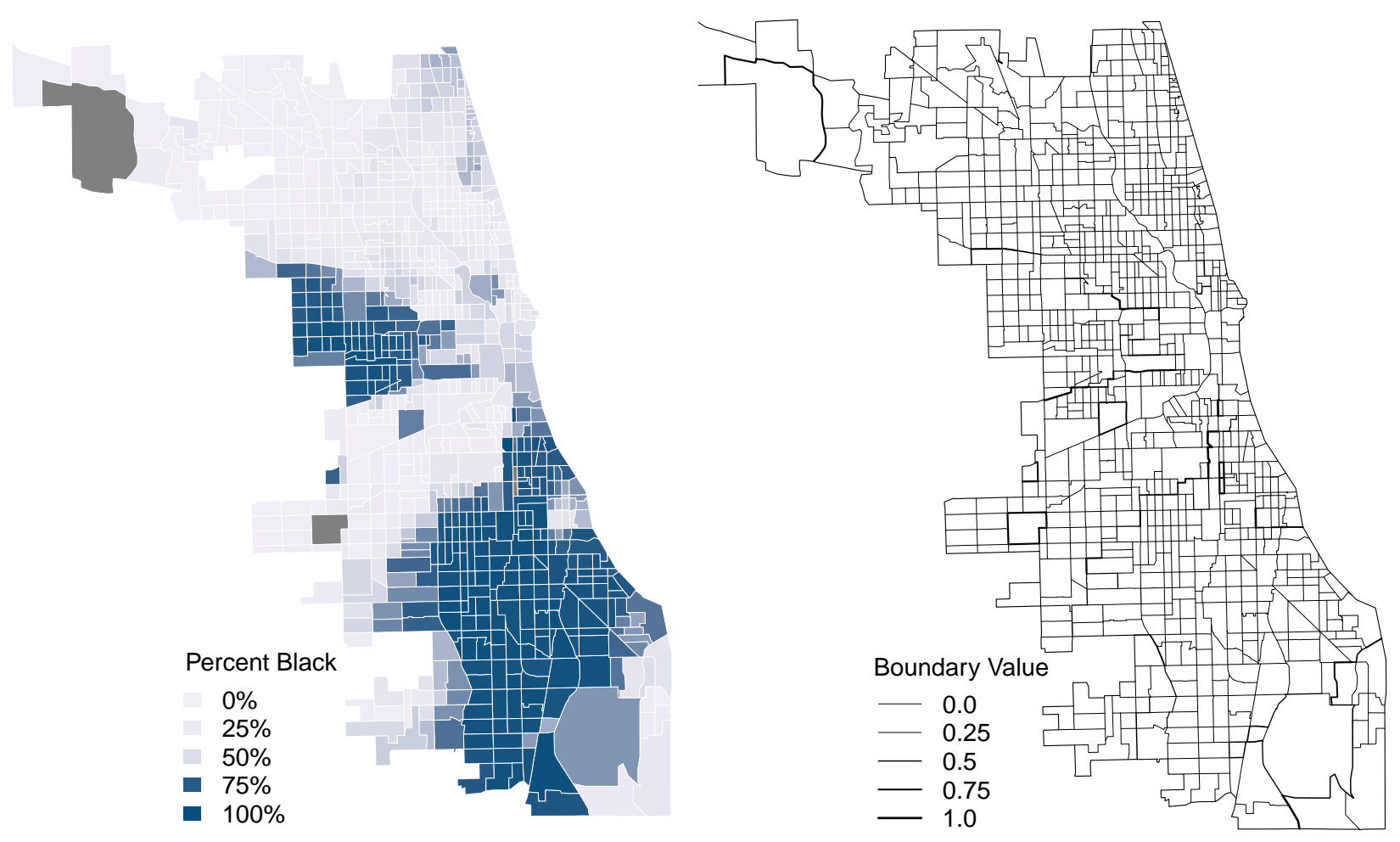

The boundary likelihood value or boundary value refers to a pair of adjacent regions or rather the border line segment between two areas. Census data, however, is available for areal units (census blocks, block groups or tracts). To address this problem, we define the boundary value for an areal unit as the maximum boundary value between the focal areal unit and its neighbors.

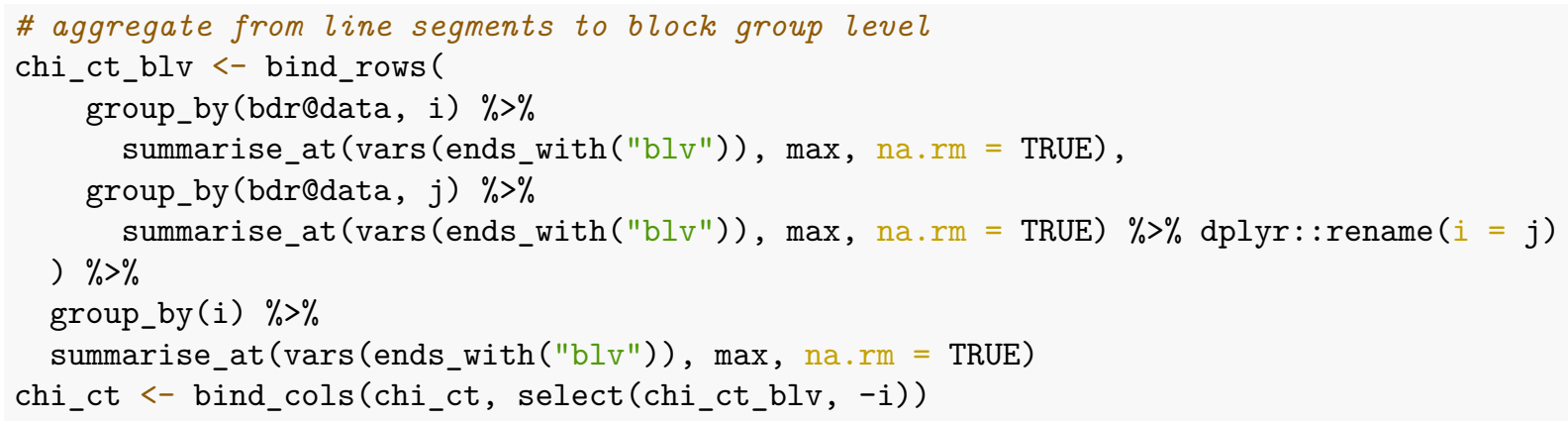

As a result, we obtain boundary values for each areal unit (census tract). Let's compare the boundary values for spatial lines (border line segments) with the boundary values for areal units.

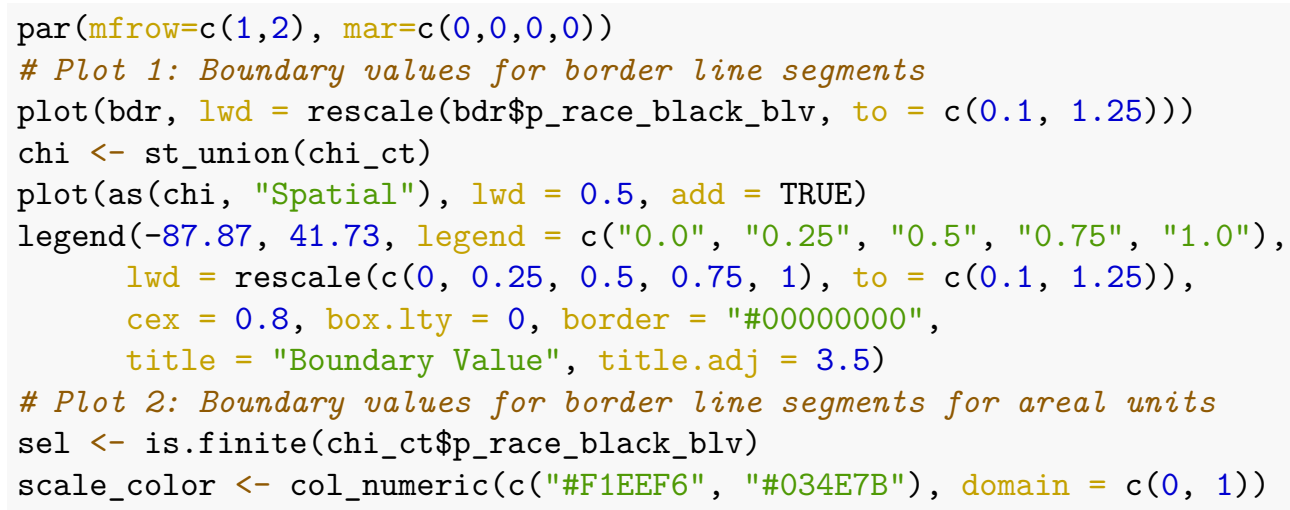



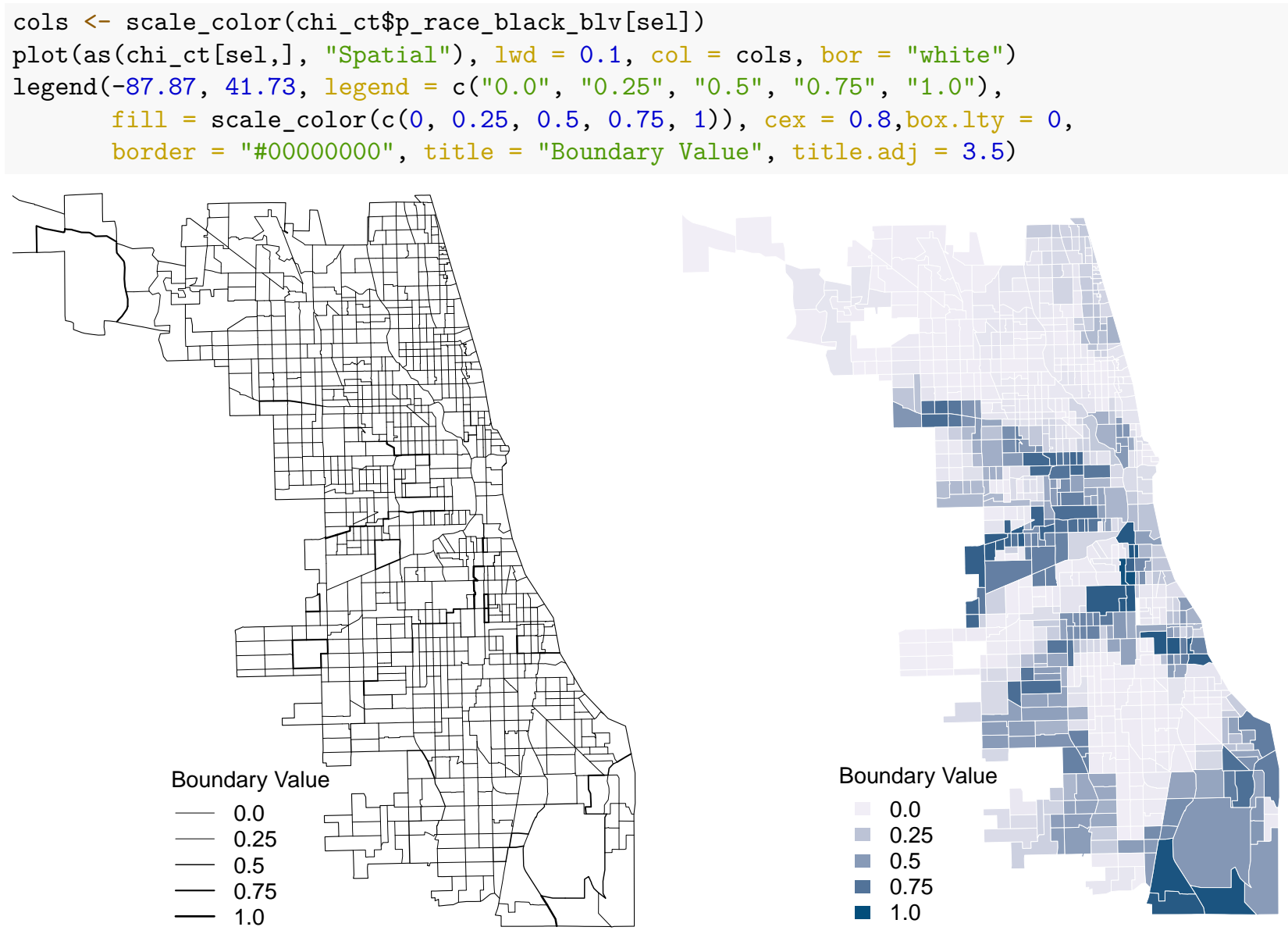

These boundary values are based on the change in a single response variable across areas (e.g. proportion African-American) but do not account for multi-group settings. Legewie (2018) derives two measures from the boundary values that are appropriate for multi-group settings. The first is a composite measure "neighborhood boundary' that combines the boundary values across different racial groups. This measure is defined as the maximum boundary value for a specific areal unit across the different race-specific values for proportion white, African-American, Hispanic and Asian. The second are pairwise boundaries between two groups such as white-black, white-Hispanic, black-Hispanic and potential others. These pair-wise boundaries are defined by the product of the two race-specific boundary values. We calculate the two measures here.

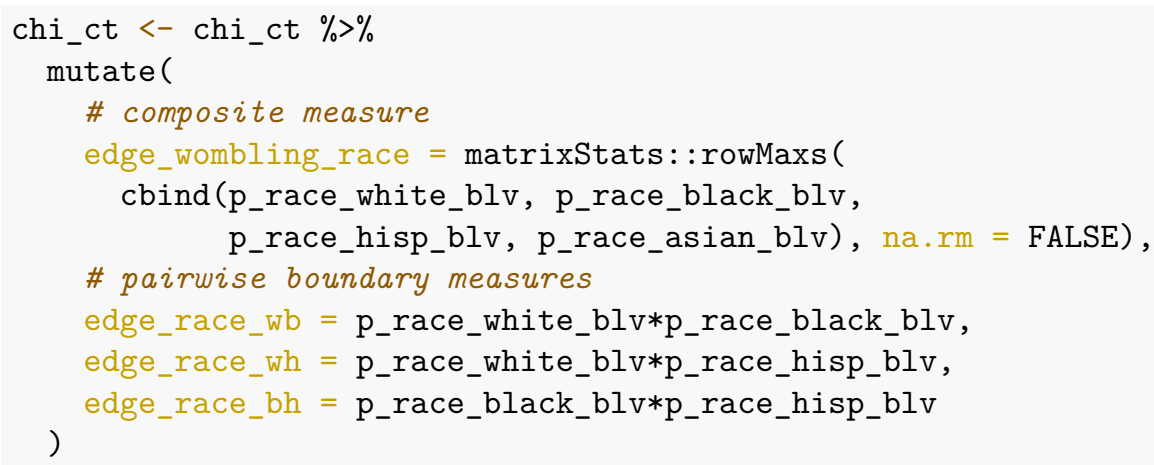




\section{Regression Models}

We model violent crime as a function of neighborhood boundaries conditional on neighborhood characteristics. The models are similar to Table 2 in Legewie (2018) but simplified and use a different level of analysis (census tracts and not blocks). They do not account for uncertainty in ACS data, ignore spatial autocorrelation and other factors. Nonetheless, they illustrate the integration of boundary measures in standard regression settings. The first model shows the relation between the composite measures and violent crimes. Similar to Legewie (2018), the point estimate for edge_wombling_race is positive. Importantly, however, it is not statistically significant potentially because of the different level of analysis ( 807 census tracts compared to more than 36,000 census blocks).

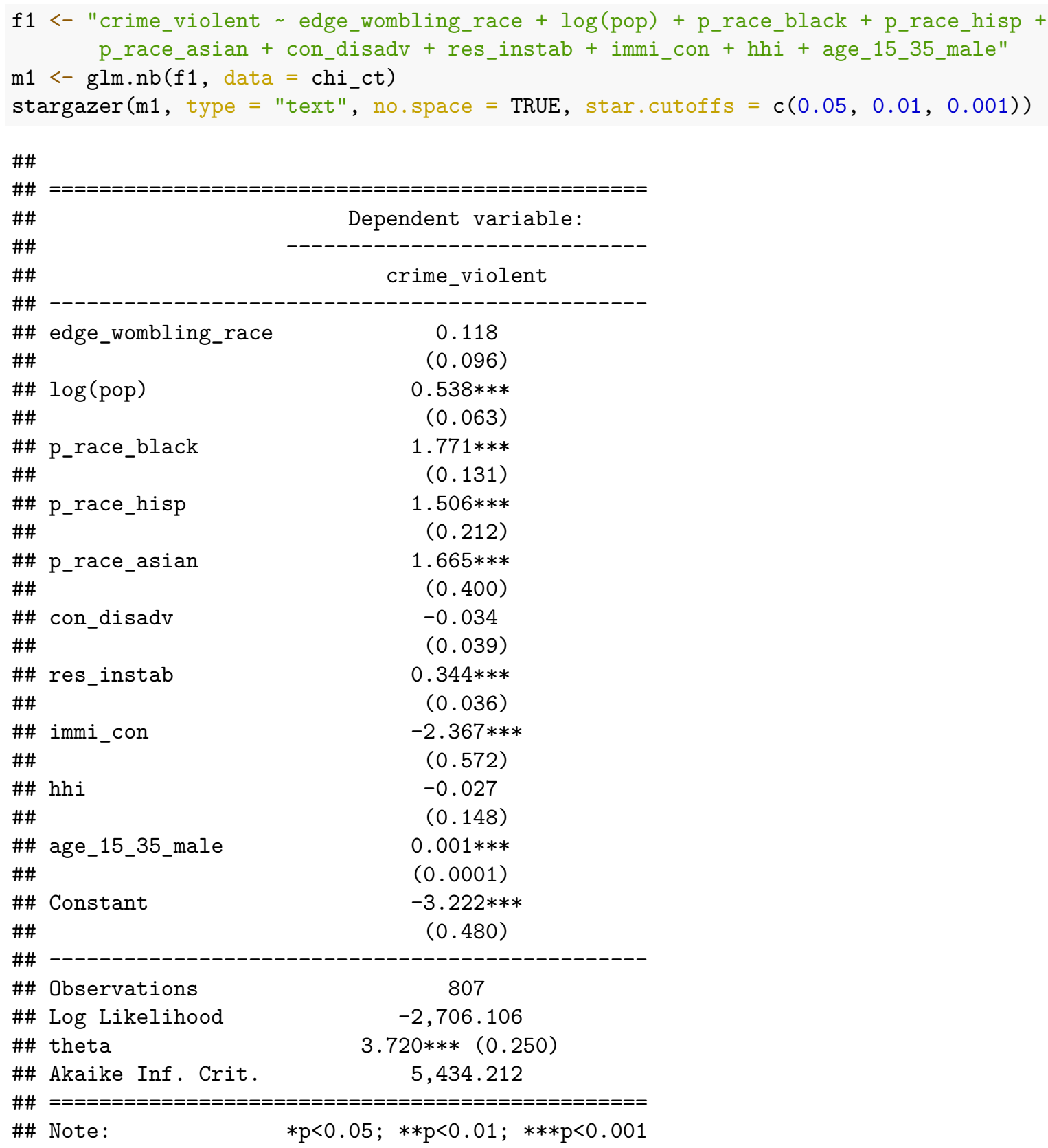

Finally, we compare the composite measure to the pairwise boundary measures for white-Black, white-Hispanic, and Black-Hispanic. 


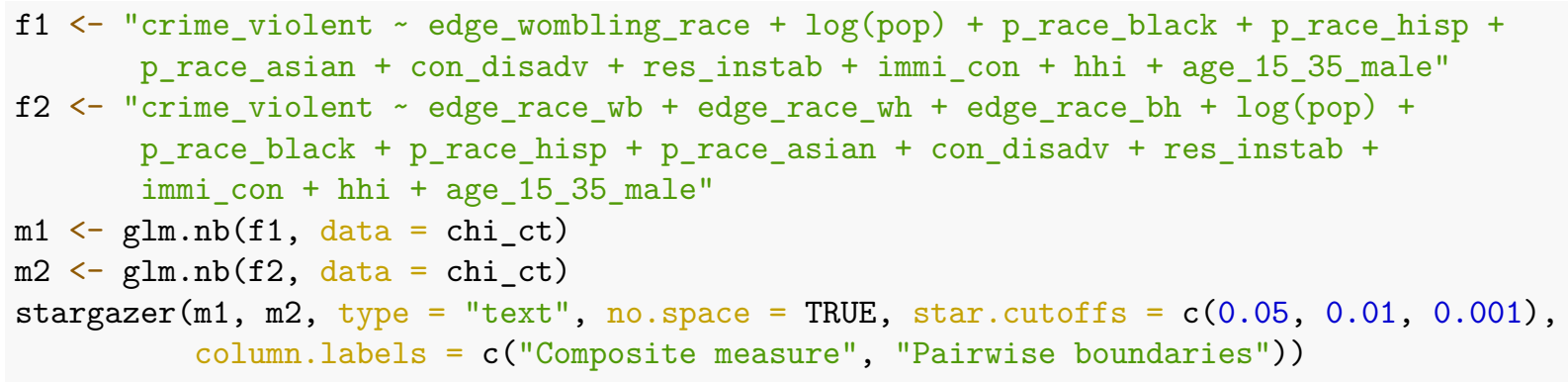




\section{References}

Legewie, Joscha. 2018. "Neighborhood Boundaries and the Spatial Dynamics of Violent Crime." Demography 55 (5): 1957-77. 